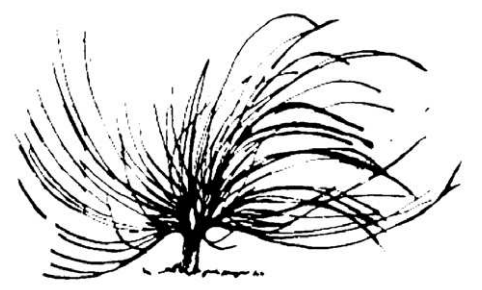

\title{
Interés por la Pedagogía Crítica: ideas sobre unas articulaciones
}

\author{
Mauricia D' Antoni ${ }^{l}$ \\ Universidad Nacional de Costa Rica \\ Heredia, Costa Rica \\ maurizia.dantoni@gmail.com
}

\begin{abstract}
Resumen
Parto de la constatación de que en Costa Rica existe un renovado interés para la Pedagogía Crítica, con ejemplos concretos. Luego trato de indicar cuáles son los pensadores y pensadoras que aportaron a la construcción de esta pedagogía o ayudaron a construir la propuesta. La reflexión está enlazada con la constatación de una crisis en la educación costarricense: la pedagogía crítica podría indicar un camino para la reforma educativa y el cambio en favor de poblaciones excluidas de la educación. Por esta razón, veo el interés por la pedagogía crítica como un indicador de la esperanza y del deseo de renovación que está vivo a pesar de estos tiempos difíciles.
\end{abstract}

Palabras clave: pedagogía crítica, alfabetización crítica, educación en Costa Rica, influencias de la pedagogía crítica.

\begin{abstract}
I start from the observation that in Costa Rica, there is a renewed interest in Critical Pedagogy with concrete examples. Then, I try to find out about which thinkers helped to build Critical Pedagogy and those who helped build the
\end{abstract}

Recibido: 28 de noviembre de 2012 - Aprobado: 11 de octubre de 2013

1 Docente catedrática en psicología social e investigadora en la Escuela de Psicología y en el Departamento de Docencia Universitaria de la Universidad de Costa Rica. Es investigadora en el Proyecto Alfabetización Crítica y Derechos Humanos de la División de Educología de la Universidad Nacional. 
proposal. This reflection is linked to the finding of a crisis in the Costa Rican education: Critical Pedagogy could indicate a path to educational reform and could help excluded population. For this reason, I see the interest in critical pedagogy as an indicator of hope and desire for renewal that is alivedespite these difficult times.

Keywords: critical pedagogy, critical literacy,education in Costa Rica,influences of critical pedagogy

\section{Explorando la pedagogía crítica en Costa Rica}

$\mathrm{D}$ urante el primer y segundo semestre del año 2012, varias compañeras de la Universidad de Costa Rica y de la Universidad Nacional, se me han acercado pidiéndome intercambiar ideas acerca de pedagogía crítica.

Sobre este particular, menciono que las secciones de Educación Primaria y de Educación Preescolar de la Escuela de Formación Docente de la Universidad de Costa Rica recientemente, en diferentes momentos y enfrentando diferentes etapas del proceso, la decisión de orientar sus procesos de autoevaluación o de revisión curricular hacia planteamientos informados por el enfoque en objeto

El Departamento de Docencia Universitaria, de la Universidad de Costa Rica, ha puesto en agenda un conversatorio sobre Pedagogía crítica que servirá, entre otros, como momento de formación del equipo docente de esta unidad académica..

La División de Educología del Centro de Investigación en Docencia y Educación de la Universidad Nacional (UNA), donde me desempeño como investigadora, también ha alimentado su revisión curricular con este enfoque pedagógico, a la vez, está planificando acciones para la capacitación del cuerpo docente y el intercambio de ideas en el ámbito educativo.

Finalmente, la Asociación de Estudiantes de Educación Básica (DEB) en la Universidad Nacional realizó un taller acerca de Pedagogía crítica, en el marco de la semana de Educación Básica de esa Universidad, que ya se impartió. Deseo aclarar que la DEB es la instancia que forma a las futuras maestras de primaria en esa universidad.

Intrigada y complacida por este renacer del interés dirigido a tal experiencia del pensamiento sobre educación, declaro que muy evidentemente no soy una experta en el tema, sino una apasionada del mismo 
y como tal quisiera proponer un pequeño recorrido entre algunas de las ideas que la Pedagogía crítica ha dado a conocer, vinculándolas con dos temas: la pedagogía de Paulo Freire y la alfabetización crítica.

Quisiera discutir cuáles son, en mi opinión, las coincidencias entre tales cuestiones teóricas en un contexto costarricense, sin olvidar los tremendos retos que la institución educativa está enfrentando en estos momentos.

Es evidente (III Informe del Estado de la Educación Costarricense, 2011) que la institución educativa en Costa Rica atraviesa una grave crisis, desde el nivel preescolar e incluye la Universidad. Las razones de la crisis son diferentes y su exposición merecería una larga discusión aparte. Las condiciones políticas y estructurales del país son sin dudas un ámbito de estudio que merece ser estudiado y vinculado con la crisis educativa, ya que proporcionaría muchos insumos para ese vasto análisis (Gómez \& Mora, 2012). Las universidades costarricenses han realizado un gran volumen de investigaciones y debates que muestran la existencia de una conciencia difusa sobre la existencia de una crisis educativa, a pesar de que tengamos diferentes ideas sobre sus causas y posibles soluciones.

Reitero mi sorpresa al darme cuenta que varios compañeros y compañeras que trabajan hoy en la academia costarricense tienen interés no sólo en conocer más acerca de Pedagogía crítica sino también utilizar esas ideas para construir capacitación, curricula y nutrir propuestas pedagógicas. Lo anterior nace del supuesto de que el interés manifestado en la Pedagogía crítica arranca de la esperanza, del deseo de cambio. Me atrevo a asomar la hipótesis de que los y las docentes de las dos universidades nacionales que he encontrado alrededor de la temática han madurado la idea de crisis y han sustituido esa noción por la de cambio. Todavía ese cambio no tiene dirección segura, pero puede ser que la inseguridad sea justamente el camino que andamos buscando.

\section{Algunos Nutrientes de la Pedagogía Crítica}

Me propongo buscar algunos nutrientes de esta pedagogía: empiezo con decir que, al compartir con compañeros y compañeras momentos de debate sobre Pedagogía Crítica he sentido a veces la necesidad de aclarar de una vez que sus raíces son marxistas (McLaren, 1988), para evitar de desnaturalizar, como se verá más adelante, una 
propuesta que encuentra de su núcleo constitutivo más profundo y aglutinador en una posición filosófica y política clara.

En cuanto a sus orígenes, podría adelantar que se ha definido a la Pedagogía Critica como una aproximación pedagógica que trata de ponerse preguntas acerca de los procesos de dominación y de prácticas, costumbres, supuestos, mitos, construcciones sociales, ideologías dominantes, a favor de todos los actores y actrices del proceso formativo ${ }^{2}$ en el ámbito institucional.

La pedagogía crítica rechaza la división entre cultura superior y cultura popular, dándose así a la tarea de rescatar los saberes cotidianos, el conocimiento que se deriva de las historias vividas por las personas. Como se verá en el documento y con la referencia de Freire, emerge la dimensión ética como central como momento irrenunciable en el proceso educativo. En este sentido, la Pedagogía crítica, en su análisis de las normas sociales y culturales, en su dejarse perturbar por las desigualdades, el tema del poder, en su centrarse en la diversidad, escoge una dimensión ética para su quehacer.

Con Giroux (1997) y obligando toda esta rica tradición investigativa a caber en una definición, se podría decir que la pedagogía crítica se enfoca en crear nuevas formas de saber, insistiendo en la construcción de las divisiones entre disciplinas, creando saberes interdisciplinarios. Trata además de formular preguntas sobre las relaciones entre lo que llama "las periferias", opuestas a los centros de poder, en las escuelas, en las universidades, en la educación en general. La pedagogía crítica se propone construir un proyecto pedagógico político a partir de una lectura de la historia a fin de abordar asuntos de clase social, de raza y de género.

La pedagogía crítica puede ser vista también como un proceso pedagógico con miras al desarrollo de una conciencia crítica. Como concepto ha involucrado a un grupo de investigadores de todo el mundo, tal es el caso de O 'Sullivan (1999), el cual ha afirmado que el origen del concepto se debe a La universidad de Toronto, y allí al Ontario Institute for Studies in Education. Ciertamente la inspiración para esos estudios se la debemos a la Pedagogía del Oprimido de Paulo Freire, a

2 En este contexto hablo de "proceso formativo" ya que expresiones como "proceso de enseñanza y aprendizaje" o "actividad de aula" sería reductivas, por no tomar en cuenta la red de relaciones y el entramado de acontecimientos significativos para el aprendizaje y la construcción de sí de la persona en el contexto institucional - educativo. 
la Escuela de Frankfurt, y, entre los filósofos de la teoría crítica, especialmente a Habermas (1988).

Foucault (2000) estudia temas como verdad, poder, control, instituciones, entre ellas la escuela. Él es uno de los teóricos - guía para la Pedagogía Crítica, con una influencia sobre McLaren (1988), que el investigador canadiense declara.

También le debemos gratitud a John Dewey por sus conceptos sobre educación y democracia (Mayo, 2011), mientras que Antonio Gramsci enriquece la herencia de la pedagogía crítica con sus estudios sobre educación y hegemonía. Es Giroux (2003), quien retoma a Gramsci, oponiendo el pensamiento del filósofo italiano a intentos conservadores de relectura en los Estados Unidos.

Autores como Williams (1983), al igual que antes que él Gramsci (cit. Por Giroux, 2003a), o que después Freire (2001; 2005), comparten el siguiente enfoque: un aspecto fundamental para la educación es el de las representaciones culturales, es decir, el esquema mental que nos hacemos de la realidad. Esta realidad marcada y construida para pensar de una determinada manera, se encuentra en las construcciones simbólicas imperiales que delimitan la realidad completa que se constituye en las relaciones entre las mujeres y los hombres.

Entre las personas exponentes en la contemporaneidad, que probablemente se encontrarían a gusto bajo esta bandera, se pueden mencionar (Mayo, 2011) Corrigan (1990), Lather (2007), Shor y Parr (1999), hooks (1981, en minúsculas por deseo de la autora), Britzman, citada por Mayo, 2011) y el ya citado O 'Sullivan.

Es necesario subrayar que Paulo Freire ha sido vinculado con la temática de la pedagogía crítica; según algunos es uno de sus padres. Independientemente de eso, Freire ha sido y es el inspirador de mucha parte del trabajo de la pedagogía crítica, no solamente en América latina. Además de él, entre personas ya fallecidas, le debemos ideas a teóricos como el afroamericano Dubois (nacido en 1868 y fallecido en 1963), (1935); Gómez (nacido en 1952), de la Universidad de Barcelona, amigo de Freire y estudioso del amor como fuerza política (2006) y, finalmente, Joe Kincheloe (nacido en 1950 y fallecido en 2008); con quien colaboró, entre otros, Steinberg (2000).

Considerado uno de los estudiosos de referencia para la pedagogía del constructivismo de la educación multicultural, críticos del debate contemporáneos sobre curriculum, Kincheloe (2001) fundó en 
Canadá el proyecto Internacional Paulo y Nita Freire (The Paulo and Nita Freire International Project for Critical Pedagogy) en la Facultad de Educación de la Universidad McGill. El objetivo era crear una comunidad global de investigadores y personas involucradas en la acción social bajo la bandera de la pedagogía crítica. Uno de los conceptos que le debemos a Kincheloe (2001), es la teoría cognitiva crítica, que se enfoca en investigar relaciones de poder para ir construyendo una psicología de la educación más amplias y liberadoras: el fin es el de desarrollar una psicología de la posibilidad. Este proyecto tiene como meta la de de avanzar en la propuesta freiriana y llevar la pedagogía crítica a una "segunda fase" de su evolución. La idea es la de construir un movimiento decolonizador mundial, dedicado a la escucha y el aprendizaje de gente de todo el mundo y de discursos diferentes. Los conocimientos indígenas son uno de los nutrientes de esta segunda pedagogía crítica (Kincheloe y Steinberg, 2000), con la potencialidad de ensanchar la pedagogía crítica y llevarla hacia la cuestión de la hegemonía en educación.

Naturalmente el marxismo y la teoría crítica de la Escuela de Frankfurt han sido los nutrientes fundamentales para la propuesta de la pedagogía crítica, pero entre los influjos que han aportado a la construcción de esta pedagogía, hay quien menciona (Mayo, 2011) los estudios culturales y los estudios poscoloniales y a Said (1990) en un campo de la educación donde asistimos a esfuerzos de diversificación de los procesos de formación que todavía están lejos de alcanzar las finalidades de una educación ciudadana, a pesar de tratar de innovar. Se alerta en contra de la creación, por parte de fuerzas imperiales de una única identidad cultural homogénea que, sin embargo se trata de "vender" como diversa y uniforme (Guimarães, 2009).

Otro nutriente de la pedagogía crítica es el feminismo y la teoría de género, así como la teoría crítica sobre raza y etnicidad y aquí podríamos citar nuevamente a Said (1990) y a Leather (2007). Esta última propone formas de autoridad en la educación que sean dialógicas, pluralistas, non dualistas y anti jerárquicas. Estas nuevas formas de autoridad se redefinen como formas del conocimiento legítimo en el posmodernismo de la resistencia. 


\section{Giroux y el papel docente}

Giroux es uno de los teóricos que a mi parecer más se conocen en Costa Rica. Su importancia es determinante y me parece muy urgente resumir algunos de sus hallazgos. Central es la necesidad de apropiarnos de la reflexión que él hace sobre el rol de los y las educadora en el mundo actual y su relación con la institución escolar (Giroux ,1983).

Giroux $\left(2003^{\mathrm{a}}\right)$ investiga también la mercantilización que asecha a la institución escolar, no sólo en términos de la dicotomía escuela pública y privada, sino mirando también adentro de la institución misma. Es este un peligro presente también en Costa Rica.

Para Giroux (1997; 1997a), la tarea del educador crítico es la de cartografiar el modo en que las ideologías se inscriben en las relaciones de la escolarización. El curriculum es otro territorio para el análisis docente, así como las formas de organización educativa. Sumamente importante es también la reflexión sobre la relación entre docentes y el estudiantado, en particular, ver cómo las ideologías son asumidas por los y las estudiantes y como dan cuerpo a sus sueños, deseos e ideas subjetivas.

Me parece muy atinada también la propuesta que se le hace al cuerpo docente de transformarse en intelectuales transformativos (Giroux, 1997a ; 2003). Creo que los y las docentes acumulan en su trayectoria profesional una gran cantidad de información, de datos que, de manera más o menos ingenua, ellos y ellas recopilan para confeccionar teoría. Esta teoría es lo que les permite actuar en su salón de clases y en la institución.

Puede ser que ellos y ellas no procesen esa meta teoría, ni sean todavía capaces de vincular lo que ven en el acontecer cotidiano en su aula con la "teoría académica", la que está en los libros. Sin embargo, sería muy difícil llevar a cabo una práctica profesional sin elaborar lo vivido, sin organizar, sacar conclusiones, categorizar lo que se experimenta y con todo ese material orientar el quehacer cotidiano. Interesante es el estudio de como la "teoría ingenua" derivada de la práctica en el trabajo se relaciona con el conocimiento adquirido durante la formación académica y a través de las capacitaciones.

El peligro que enfrentan los y las docentes puede ser otro: el de la alienación, si sucumben a los mandatos de la ideología dominante, si dejan de leer lo oculto de los curricula y no se ubican a la par de sus 
estudiantes, revisando de manera crítica los intentos de la reproducción social y cultural a través de la institución escolar $(1997 ; 2001)$.

El trabajo de Giroux es crítico y a la vez propositivo. A la vez que se preocupa por las formas de reflexión autocrítica que los y las docentes tienen que alcanzar para modificar su rol tradicional, impulsa el una praxis capaz de transformar reflexivamente la base de los conocimientos que se imparten y se opone a presupuesto elitistas, excluyentes, injustos en campo educativo (Giroux, 1998).

\section{Michael Apple y Peter McLaren}

Otros dos teóricos destacados de la pedagogía crítica son Apple y McLaren. Deseo describir brevemente algunos momentos de sus propuestas que me parecen relevantes para este ensayo en la contingencia educativa que Costa Rica atraviesa.

La modernización conservadora de Apple (2002) apela al "sentido común" y reelabora el significado de conceptos clave para entender el mundo actual y en él la educación: mercado, niveles, religión, desigualdad. Para él el papel de historiadores y críticos sociales consiste en ser una memoria profesional para los ciudadanos que a veces desean olvidar. Aquí la referencia es a EE.UU, donde Apple vive, pero es fácilmente vinculable con la problemática de América Latina. En cuanto a mercados y conocimiento, Apple nos alerta para que revisemos con cuidado la lectura que los teóricos conservadores hacen del "fracaso total de las instituciones educativas", oponiéndose al igualitarismo. Existen grupos y clases (neoliberales, neoconservadores, populistas autoritarios, clase media directiva y profesional) que se unen para crear una nueva cultura conservadora de la educación y el autor describe estos grupos y destaca sus contradicciones, analizando la situación como el autor la percibe en los Estados Unidos.. Dice que existen nuevos burgueses (nueva clase media directiva y profesional) ideológicamente no alineados con la derecha, que sin embargo buscan mayor control educativo y la instauración de niveles educativos más elevados con el fin de elevar el nivel educativo de sus hijos. En la escuela pública estos últimos partirían así con un capital cultural mayor en la competencia con la juventud de las clases más desposeídas. De estas reflexiones, surge el temor que Apple (2002) expresa para el futuro económico de los hijos de clases excluidas. 
Con respecto a los indicadores y los ranking que ahora están tan de moda y que forman la "imagen pública" de un colegio, Apple (2002) opina que son importantes y también lo son las propuestas de evaluación; la evaluación de la enseñanza utilizando los resultados de las pruebas nacionales, etc. La propuesta es la de crear movimientos fuertes que elaboren y defiendan políticas más progresistas. Claro que existen peligros, en cuenta la debilidad de los sindicatos de los maestros, su orientación política, la falta de consenso sobre los objetivos de una educación progresista: el comentario es una vez más relativo a los Estados Unidos, pero puede ayudarnos a reflexionar sobre nuestra realidad, que no es muy distante de la de los vecinos del norte.

Los movimientos progresistas para "replantear la escuela" no tienen recursos ni apoyos o gran capacidad de movilización. Apple (2002) cree que existen esperanzas, aún cuando se ve que la solución es ejercer mayor control sobre el currículo y la enseñanza. La entrevista a los padres destaca los temores y aciertos que Apple ha expresado acerca de las desigualdades educativas y el autor le dedica finalmente atención a la concentración de poder de las estructuras administrativas. Pobreza, poder político y económico explican mucho mejor las iniquidades del rendimiento escolar que otras variables.

McLaren es un teórico canadiense de la pedagogía crítica bien conocido en Costa Rica. En su trabajo el acento es puesto en la centralidad de la política y del poder. Para conocer el funcionamiento del sistema educativo y de la escuela es esencial tener en la mira la economía política de una educación en un Estado. Le interesa el tema de la construcción de subjetividad en el estudiantado. La persona joven está en proceso de construirse a sí misma, por lo tanto, las influencias, los modelajes docentes, el papel del medio son todas aristas importantes en el proceso de desarrollo. El autor tiene en la mira el análisis crítico de la textualidad, entendida esta última como toda la producción discursiva en ámbito educativo: más allá del conocido "curriculum oculto", hay mucho que se dice y que se imprime para realizar el trabajo de clase y alrededor de este. (McLaren, 1994; Giroux \& McLaren, 1994). Se trata de materiales que hay que tratar con suspicacia y de manera crítica. McLaren (2007) tiene la característica de escribir libros en forma de diario, utilizando la investigación - acción y la observación etnográfica para dar cuenta de lo que sucede en el campo educativo y para alertar sobre la responsabilidad adulta con respecto a la inequidad en la 
educación. En cambio, los y las educadoras tenemos la responsabilidad de generar una pedagogía que vaya más allá de la pura reproducción del conocimiento, una pedagogía que potencie la moralidad en las relaciones sociales. Para él, esta es la verdadera esencia de la pedagogía crítica, que debería ser revolucionaria y capaz de potenciar la resistencia contra la opresión.

McLaren, (2007) al igual que bell hoks (2003, quien escribe su seudónimo sin mayúsculas), llevan su crítica a la influencia de las instrucciones, de las estructuras sociales, de la globalización y los medios de comunicación, las relaciones de raza y se interesan por la temática de la resistencia y de las posibilidades de cambio.

\section{Freire y la Pedagogía Crítica}

Freire es visto como un punto de referencia obligado para la Pedagogía crítica: este apartado tiene la finalidad de manifestar qué tanto le debe esta corriente de pensamiento educativo al educador y filósofo brasileño.

Rodríguez (2010) le atribuye a Freire la enunciación como propuesta de acción de la pedagogía crítica, ubicando un proyecto ya maduro en este sentido en su Pedagogía del Oprimido (Freire, 1970). La oposición que encontramos en Freire es entre pedagogía bancaria y pedagogía crítica: los que se consideran poseedores del conocimientos conciben una educación acumulativa, los y las que son vistos como ignorantes absolutos son las personas encaminadas a construir la educación liberadora en una sociedad más humana y más justa (Freire, 1970).

La pedagogía crítica nació leyendo a Freire, tal vez el educador crítico más conocido, visionario, inspirador en el panorama educativo reciente en América Latina.

Un mismo origen marxista vincula a Freire y a la Pedagoga a crítica: Si Marx (en Masi, 2008), subraya el rol activo del sujeto en la construcción del conocimiento, por interpretarlo como un resultado de un cruce de determinaciones sociales (en particular la relación entre producción y trabajo), Freire recuerda que el ser humano es capaz de alejarse del objeto para admirarlo, puede "objetivar" y tomar así distancia frente al mundo. La praxis humana es actuar de manera consciente sobre la realidad objetiva.

En el plano educativo, Freire le atribuye al estudiantado la capacidad de pensar de manera crítica, situación que les permite reconocer 
relaciones entre los problemas sociales que enfrentan y el contexto social el que están insertos(as). El concepto de concientización describe este proceso de tomar conciencia y representa el primer paso de la praxis (Masi, 2008). De allí el proceso de transformación social, producto de la praxis colectiva. En efecto, la reflexión y la acción son unidades indisolubles: si no fuera "praxis" sería activismo vacío, sería señal de equivocación en captar la realidad. Freire luchó para lograr un proceso de construcción de una subjetividad democrática, advirtiendo explícitamente los riesgos de la ideología neoliberal con su fatalismo, un pecado del qué cuidarse ya que atenta contra la autonomía de los seres humanos. (Masi, 2008). La propuesta de Freire es inspiradora y esperanzadora (Freire, 2005), él cree en la resignificación de la utopía como proyecto que se concreta en la praxis.

Freire ha hablado de utopía, esperanza y concretud al igual que la pedagogía crítica, y en algunos casos a la par de esta, llevando la teoría a desarrollos y aplicaciones actuales. Esta fue una puerta que Freire dejó abierta al hablar de problematización.

Muchos pedagogos críticos de la actualidad se han interesado por el posmodernismo, perspectivas antiesencialistas del individuo, del lenguaje y han estudiado el poder. Se ha mantenido el interés freiriano en la crítica a los gobiernos opresivos y a la relación poder/ conocimiento en relación con el cambio social.

\section{Pedagogía Crítica ante los grandes retos para la educación pública}

Me parece importante enlazar el tema del ensayo con la situación que enfrenta la educación actual en Costa Rica. En efecto, el momento que vivimos inspira inquietudes: está la crisis de la educación, pero antes de la crisis encontramos el peligro que la educación pública corre ante los empujes del modo de producción capitalista y hacia la mercantilización de bienes públicos como la educación (Meoño, 2011).

Pienso que la pedagogía crítica, más que dar respuesta a los temores que embargan quienes creemos en una educación con una finalidad liberadora, nació para posicionarse como alternativa a los modelos educativos finalizados al consenso, a la reproducción social y cultural (Morrow \& Torres, 1995).

Asistimos en la contemporaneidad a un complejo proceso de desarrollo de la sociedades latinoamericanas que ha implicado 
profundas transformaciones en las relaciones sociales: la imposición del modo de producción capitalista, hoy largamente dominante, subordina la economía en los países periféricos, como lo son los latinoamericanos (Meoño, 2011).

En este contexto, el sistema educativo forma parte de procesos de mercantilización, al igual que otros productos, servicios, "cosas". Giroux $\left(2003^{\mathrm{a}}\right)$ identifica esos procesos de mercantilización en el interior de los colegios.

Los Estados nacionales y los organismos internacionales están desarrollando un papel central en estos procesos, impulsando políticas públicas económicas y sociales, procesos inevitablemente integrados, en alianza y en confrontación con diferentes sectores sociales en los ámbitos nacional y transnacional. En efecto, por medio de políticas impuestas por organismos financieros internacionales, los gobiernos pueden crear leyes, abrir y cerrar instituciones o programas, firmar convenios dentro o fuera de los países, asumir compromisos económicos y políticos, impulsar o eliminar programas y servicios.

Muchas veces nos presentan estas iniciativas como "reformas necesarias"; entre otros advertimos, por parte de las escuelas y de las universidades (públicas) la creciente tentación de medirse y compararse a través de ranking, de "autoevaluarse", "acreditarse", siempre haciendo referencia a rúbricas construidas en ámbitos cercanos a organismos internacionales.

Mientras se discute de la necesidad de realizar una gran reforma educativa, un problema que se siente en varios países de América Latina, es posible sucumbir a la fascinación que provocan las comentadas iniciativas en pro de la "excelencia educativa" (McLaren, 2005).

En Costa Rica, una "reforma educativa" que se ve como urgente (CONARE, III Estado de la Educación, 2011) se inició con el apoyo de una (o varias) empresas privadas de consultores, sin tomar en cuenta en la construcción de esta propuesta a los actores y actrices del contexto educativo: docentes, estudiantado, sector administrativo, sindicatos, padres y madres de familia.

Acerca de la crisis educativa que mencioné, que involucra toda América Latina (Gvirtz \& Beech, 2008) algunos aspectos de la situación educativa que se consideran más alarmantes son la exclusión del ámbito educativo (que en los textos aparece a veces como "deserción escolar"), la falta de motivación del estudiantado (no hay mayor explicación sobre 
el particular), las dudas de estudiantado y docentes acerca de la oferta académica (CONARE, II Estado de la Educación, 2008). Otros problemas graves involucran a los curricula, el autoritarismo (D' Antoni \& Gómez, 2011; D' Antoni, Gómez, Gómez \& Soto, 2011) y la violencia en las aulas, que los medios de comunicación tienden a leer de forma tal vez superficial y donde el bullying (matonismo) es tema que amerita mayor interés de la violencia estructural donde está, de alguna manera, insertado.

Es urgente que uno de los emergentes que atraen la atención de quienes analizan la situación escolar, conceptualizado tradicionalmente como fracaso escolar, se contextualice de manera situacional. Baquero (2001) nos propone una lectura del llamado "fracaso escolar" como problema psicoeducativo. Baquero profundiza la definición de las condiciones o aspectos de la educabilidad, entendida como la delimitación de las condiciones, alcances y límites que posee potencialmente la acción educativa sobre sujetos definidos en situaciones definidas. Las concepciones presentes sobre la educabilidad de los sujetos se vinculan de modo bastante directo con las razones que se atribuyen al fracaso escolar masivo, con la caracterización del dispositivo escolar "común", pero también, naturalmente, con la necesidad de diseñar ofertas educativas y estrategias integradoras (Baquero, 2001).

La teoría de Lev Vygotski interesa porque rehúye de esquemas mecanicistas en el desarrollo de la persona joven y enfatiza que la comprensión del desarrollo y del fenómeno educativo sólo se puede dar a partir de la comprensión del contexto y del ser humano como actor de sus procesos.

A finales de los años 80 y 90, impulsado también por traducciones de parte de su obra al español, se da un redescubrimiento del psicólogo ruso Lev Vygotski, que interesa al subcontinente americano también por sus articulaciones con la pedagogía crítica y con la pedagogía del oprimido de Paulo Freire. Actualmente Greca (2005), Baquero (2001), Fariñas León (2006), González Rey (2007), Rodríguez (2008), Terigi (2009), junto con muchos otros, son académicos y académicas de varios países de América Latina que estudian a Vygotski y realizan propuestas con particular atención a la problemática educativa propia de América Latina (Rodríguez, 2008).

Existe actualmente un proyecto aprobado en el Instituto de Investigación en Educación de la Universidad de Costa Rica, con el nombre de Actividades académicas para la consolidación de grupo investigador: 
aplicaciones de la teoría de Vygotski en contexto latinoamericano, que tiene el propósito de mantener viva esa tradición de estudios en Costa Rica y presenta estrecha relación con la pedagogía crítica.

\section{Algunas articulaciones: Alfabetización Crítica}

A continuación me gustaría buscar articulaciones entre la pedagogía crítica y propuestas como la de la alfabetización crítica como método de investigación o en la investigación - acción. Sin embargo, al tener ambas el calificativo de "crítico", es evidente la relación teórica y la conexión profunda más allá de lo metodológico. A través del concepto marxista de praxis, en efecto, no tenemos en esta área conceptual una división entre teoría, acción y su metodología, sino que varios momentos se comprenden y despliegan como un todo.

La alfabetización crítica ve la construcción de significado de los textos como un proceso de construcción social, que tiene su metalenguaje. Por ejemplo, el "discurso" es cualquier texto, película, canción, página literaria etc. Todas estas producciones humanas tiene su subtexto., el cual puede ser examinado y desconstruido, lo que interesa determinar es a quién se le da voz en ese discurso, véase el "curriculum oculto" (Patel Stevens \& Bean, 2007).

Pero aún más allá de la ocultación del curriculum, podríamos enfocarnos en lo que nosotros y nosotras necesitamos hacer (alfabetizarnos) para aprender a leer las intenciones y los signos pero también aprender que cada "texto" es necesario leerlo dos veces (D' Antoni, 2012).

Es interesante la idea de «textos» de Habermas (1988), quien plantea que la interpretación de un documento sirve para atribuirle significado, pero lo mismo no pasa con la interpretación de las acciones. Todas las ciencias, humanas o empíricas, al estudiarla trabajan en transformar la acción humana. Lo hacen ya sea que se estudie la "conducta», atomizando la acción en partes «manejables» para analizarlas, o bien, trabajando en un sentido más global, descubriendo formas de registrar la acción para reproducirla luego de alguna manera, ya sea en forma de notas de campo, fotografías o grabaciones. De este modo, la acción es convertida en "texto", pudiendo interpretarse de manera semejante a la utilizada para tratar cualquier otra forma de material textual. 
El saber relacionado con la comprensión debe ser juzgado según el significado interpretado, ayude o no al proceso de elaboración de juicios respecto a cómo actuar de manera moral, según la razón.

\section{Alfabetización crítica versus los peligros de lo puramente "técnico"}

Como todo lo que es "crítico" al igual que la pedagogía así denominada, la propuesta de la alfabetización crítica es la de leer críticamente los "textos" relativos al bagaje que denominamos "técnico", por ejemplo, el curriculum. Se evidencia que el interés técnico puede fácilmente convertirse en un elemento para el control y la manipulación.

Hay enfoques de diseño curricular que suponen que el o la docente tendrá influencia sobre su aprendiz. Este actuará según lo que se espera de una persona que aprenda lo que le hemos enseñado (Patel Stevens \& Bean, 2007). Con ese fin tenemos que controlar tanto el ambiente de aprendizaje como al educando. Esta forma de entender el curriculum tiene, claro está, su lenguaje, por ejemplo, como conocen todas las personas que encuentren un documento sobre el tema curricular, los "objetivos".

La raíz etimológica de este concepto curricular fundamental es "objetos": se nota así que nos encontramos en un ámbito conceptual donde el educando se convierte únicamente en una parte de un ambiente de aprendizaje, que no es visto como un todo continuamente interactuante.

Los y las docentes que se forman suelen estar enfocados en problemas técnicos y curriculares, sin que se les invite a utilizar una lectura crítica del aspecto educativo.

Giroux (1996) habla de una pedagogía basada en la gestión como opuesta a la pedagogía crítica y se preocupa por las presiones hacia seminarios obligatorios, momentos de encuentro para compartir técnicas, sobre todo para manipular el control y la disciplina. A pesar de que él está escribiendo desde otro país, lo que Giroux comenta, a mi parecer, es perfectamente comprensible en Costa Rica. El autor invita a enfocarse más bien en aprender a plantearse preguntas sobre los principios que subyacen a las diferentes metodologías pedagógicas y a las técnicas de investigación: nada de solamente "cómo enseñar" o "con qué libros". Más bien en esa pedagogía de la gestión Giroux ve el intento de devaluación y desvalorización del trabajo docente. 
Las personas que entran a trabajar en el colegio hoy están sedientas de directrices concretas para trabajar en sus aulas: los tiempos han cambiado, sus discentes ya no son fácilmente disciplinables, ya que las relaciones de poder están aún menos claras que antes y la autoridad (por dicha, en mi opinión) no es lo que solía hacer. Los medios digitales proporcionan, como es sabido, un mundo de información y de diversión que tiene características como el atractivo, la velocidad, los cambios continuos: menos disciplinable todavía es un joven ser humano que se aburre, que no ve reconocida como "alta" o "válida" su cultura y no la ve representada en el contexto educativo.

El o la docente, por el otro lado, no ha tenido el espacio para reflexionar acerca del nuevo papel de la autoridad sin autoritarismo y teme, más que reconocerlo como un recurso, que su educando o educanda ya tenga conocimientos porque los adquirieron en otra parte que no es la institución educativa.

En este contexto, si en el diseño curricular prevalece un interés técnico, se aboga por el control del ambiente educativo, de manera que el producto que resulte combine con objetivos preespecificados (Patel Stevens \& Bean, 2007).

Claro está que una vez establecida la base de la organización del saber en el curriculum, hay que decidir el contenido específico de las diversas áreas del saber: al menos esto es lo que "manda a hacer" la teoría del curriculum.

¿Y si los profesores y profesoras, en cambio, con todos los actores y actrices del sistema educativo fueran creadores (as) de curriculum?

La acción y la reflexión están dialécticamente relacionadas en la espiral de la investigación-acción, que es la manera de proceder más cercana, entretejida con la pedagogía crítica.

\section{Discusión}

La experiencia de trabajo que tenemos en los colegios (D' Antoni \& Pacheco, 2003; D'Antoni \& Soto, 2010; D'Antoni, 2008; D’ Antoni, Gómez, Gómez \& Soto, 2012) y en la escuela primaria (Aguiar \& D’ Antoni, 2008) ha mostrado que dentro de la percepción de crisis en la educación costarricense actual emergen, por parte de los actores y actrices de la institución educativa, algunas problemáticas más frecuentes. Uno de los temas es el de la indefensión de los y las jóvenes en unas prácticas de aula 
verticales, autoritarias, puramente disciplinarias, ante las cuales hay, por supuesto, manifestaciones de resistencia en el estudiantado.

Por otro lado, el cuerpo docente expresa malestar y confusión con respecto a su trabajo, y nuestra interpretación (D' Antoni, Gómez, Gómez \& Soto, 2012) es que se encuentra sometido a la autoridad, en una organización escolar que pretende transformarlo en quien se encargue de la reproducción cultural. Los y las docentes tratan de responder "obedientemente" a este llamado, pero surgen confusión y malestar, a la vez que, también, formas de resistencia.

Me gustaría interpretar en el interés por la pedagogía crítica con el que personalmente me he encontrado como una respuesta, tal vez una forma de resistencia ante el malestar por lo que sucede en las aulas y con nosotros los y las docentes: reproducción de patrones tradicionales autoritarios, contenidos aburridos, curriculum oculto, desatención por las culturas juveniles en las prácticas educativas. Giroux (2003) en particular tiene unas propuestas para la transformación del cuerpo docente, que hemos visto capaz de aprender y hacer ciencia desde sus prácticas de aula. La propuesta de Giroux es que los y las docentes se transformen en intelectuales transformadores. Para hacerlo se necesita del apoyo de formadores de formadores y es importante que se dé a partir de una visión de la educación que es política, contextual, ligada al momento histórico que estamos viviendo, y del lado de los y las jóvenes.

La escuela de hoy en Costa Rica demuestra indiferencia o desprecio por las culturas juveniles. Encontramos la ausencia de esas culturas en los curricula y el trabajo de aula (D' Antoni, Gómez, Gómez \& Soto, 2012), en el sentido de que lo que el estudiantado ama y / o sabe no entra a ser parte del trabajo de aula. En la insitución educativa, en cambio, se impone una renovación en cuanto a curricula y contenidos, ahora alejados de los intereses y de las necesidades de los y las estudiantes, tanto que inclusive los estudios que se ubican del lado de la oficialidad (CONARE, II Estado de la Educación, 2008) no pueden ocultar ese malestar.

La alfabetización crítica puede ser vista como un "brazo operativo" de la pedagogía crítica. Ha sido definida a la vez una metodología para aprender juntos a escudriñar en los contenidos "ocultos" de las textualidades impuestas y una plataforma para alertarnos ante las intenciones que nuestro modo de producción tiene para la educación contemporánea. 
El legado de Paulo Freire y de tantos y tantas estudiosas que lo han anticipado, secundado o recogido se convierte en una pedagogía de acción y de esperanza. A la pedagogía crítica se le ha reprochado ser una respuesta utópica a los problemas; sin embargo, en estos tiempos difíciles que nos toca vivir creo que es una de sus fuerzas, entendiendo utopía como sueño, esperanza, como lugar adónde ir, y ya sabemos que la intención no es la de llegar, sino la de seguir caminando.

\section{Referencias bibliográficas}

Aguiar Montealegre, T. \& D'Antoni, M. (2008). Taller de mediación cultural para maestros y maestas costarricenses. Reflexiones 87(1). Editorial UCR, San José.

Apple, M. (2002). Educar "como Dios manda". Mercados, niveles, religión y desigualdad. Buenos Aires: Paidós.

Baquero, Ricardo (2001). La educabilidad bajo sospecha. Cuaderno de Pedagogía Rosario, IV (9), 71-85.

Consejo Nacional de Rectores (CONARE). (2008). II Informe del Estado de la

Educación costarricense. San José, Costa Rica. Recuperado de http://rapidshare.com/ files/93314727/Informedelestadodelanacion.rar

Consejo Nacional de Rectores (CONARE). (2011), III Informe del Estado de la Educación costarricense. San José: Costa Rica.

Corrigan, P. (1990). Social Forms /Human capacities: Essays on Authority and Difference. London: Rutledge

D’ Antoni, M. \& Pacheco, X. (2003). ¿Por qué? Jóvenes ante el desorden mundial.

Universidad Nacional, Heredia, Costa Rica: EUNA.

D’ Antoni, M., Pacheco, X., \& Induni, G. (2005). Crisis en la educacón secundaria: reproducir o trasformar. Educare, 7 (1), 91-103.

D’Antoni, M. (2006). Derechos humanos de la infancia en un Trabajo Comunal Universitario. Actualidades investigativas en educación, 1. Recuperado de http:// revista.inie.ucr.ac.cr/articulos/1-2006/archivos/derechos infancia.pdf

D’Antoni, M. (2008). Nuevas propuestas pedagògicas y el papel social del docente. Ensayos pedagógicos, 4, 11-26.

D’Antoni, M., Gómez, L., Gómez, J., \& Soto, J. F. (2008). Escenarios Investigativos desde la Pedagogía Crítica: Culturas colegiales, juveniles y políticas. Recuperado de http://www.inie.ucr.ac.cr/encuentro/memoria/experiencias/escenarios.pdf

D’ Antoni, M., Gómez, L., Gómez, J., \& Soto, J. F. (2011). Taller: Problematizando a la autoridad desde la desobediencia y la resistencia en la educación secundaria en el marco del II Encuentro de Investigación Educativa: su incidencia en la realidad. Instituto de Investigaciones Educativas (INIE) de la Universidad de Costa Rica, realizado 1-3 de febrero (2011). Recuperado de http://congreso.inie.ucr.ac.cr/memoria/documentos/6/Acerca $\% 20 \mathrm{de} \% 20$ autoridad-M $\% 20$ DAntoni,J\%20Soto,L\%20Gomez,J\%20Gomez.pdf

D’ Antoni, M. \& Gómez, L. (2011). Estados de (in) defensión y posibles horizontes de rupturas: hacia una propuesta de defensoría de las y los estudiantes de 
educación secundaria realizada en el III Simposio Internacional y VII Nacional sobre los Derechos de la Niñez y la Adolescencia Entornos y prácticas de vida saludables en la niñez y la adolescencia. Conferencia los días 4,5 6 de octubre de 2011. Ciudad de la Investigación, Universidad de Costa Rica. San Pedro de Montes de Oca.

D’ Antoni, M., Gómez, L., Gómez, J., \& Soto, J. F. (2012). La escuela en cuestionamiento: Diálogos problematizadores sobre la resistencia escolar en procesos pedagógicos emergentes. Aceptado para su publicación por la Editorial Arlekin, San Pedro, Costa Rica.

D’ Antoni, M. (2012) Higher Education Teachers. Special Needs and Critical Literacy. From Discrimination to Empowerment. Scientific Cultural Institute Mandala. 12-14 de abril, Universidad de Trieste, Italia.

Dubois, W.E.B. (1935-1999) Black Reconstruction in America. Oxford University Press. Ellioth, J. (2000). La investigación - acción en educación. Madrid: Morata.

Fariñas León, G. (2005). Psicología, educación y sociedad: Un estudio sobre el desarrollo humano. La Habana: Editorial Félix Varela.

Foucault, M. (2000). Vigilar y Castigar: El nacimiento de la prisión (30 ${ }^{\mathrm{a}}$ Ed.). México: Siglo XXI.

Freire, P. (1970). Pedagogía del oprimido. Montevideo: Siglo XXI.

Freire, P. (2001). Pedagogía de la indignación. Madrid: MORATA.

Freire, P. (2005). Pedagogía de la esperanza. Buenos Aires: Siglo XXI Editores.

Gareca, S. B. (2005). Cultura, inteligencia y fracaso escolar: una tríada de complejo abordaje en la práctica docente. Revista Iberoamericana de Educación, 36/11. Recuperado de http://www.rieoei.org/deloslectores/1190gareca.PDF

Giroux, H. (1983). Theory and resistance in education: Pedagogy for the opposition. Critical perspectives in social theory. London. Exeter: N.H. Heinemann Educational Books.

Giroux, H. (1997), Cruzando límites: Trabajadores culturales y políticas educativas. Barcelona: Paidós.

Giroux, H. (1997a), Los profesores como intelectuales. Hacia una Pedagogía crítica del aprendizaje.España: Paidós.

Giroux, H. (2001). Cultura política y práctica educativa. España, Editorial GRAÓ.

Giroux, H. (2003). Pedagogía y política de la esperanza: Teoría, cultura y enseñanza. Buenos Aires: Amorrortu.

Giroux, H. (2003a). La inocencia robada. Juventud, multinacionales y política cultural. Madrid: Morata.

Giroux, H. \& McLaren, P. (1994). Between borders: Pedagogy and the politics of cultural studies .New York: Routledge.

Giroux, H. \& McLaren, P. (1998).La formación de los maestros en una esfera contrapública. Notas hacia una redefinición. En: McLaren (compilador). Pedagogía, identidad y poder. Los educadores y el multiculturalismo. Rosario, Argentina: Homo Sapiens.

Gómez, J. (2006). El amor en la sociedad del riesgo. Una tentativa educativa. Barcelona: El Rour.

González Rey, F. (2007). Investigación cualitativa y subjetividad. México: Mc Graw Hill.

Gómez, J. R. \& Mora, M. A. (2012). Pedagogía del futuro. Educación, sociedad y alternativas. En curso de publicación Ensayos pedagógicos, Heredia: EUNA. 
Guimarães, P. (2009). Educación, ciudadanía y democracia. En Rizoma Freiriano, 3, Recuperado de http://www.rizoma-freireano.org/index.php/rizoma-freireano-3-es

Gvirtz, S. \& Beech, J. (compiladores), (2008). Going to school in Latin America. Westport, Connecticut: Greenwood Press.

Habermas, J. (1988). On the Logic of the Social Sciences. Cambridge, MA.: MIT Press.

Hook, B. ( 2003). Teaching Community: A Pedagogy of Hope. London: Rutledge.

Instituto Nacional de Estadística y Censo. (2010). Encuesta de Hogares. Recuperado de http://www.inec.go.cr/A/MS/Encuestas/Encuesta\%20Nacional\%20de $\% 20$ Hogares/Publicaciones/1.\%20Cifras\%20b\%C3\%A1 sicas\%20sobre\%20fuerza\%20de\%20trabajo,\%20pobreza\%20e\%20ingresos.\%20Julio\%202010.pdf

Kincheloe, J. (2001). Hacia una revisión crítica del pensamiento docente. Barcelona:Octaedro.

Kincheloe, J. \& Steinberg, S. (2000). Contextualizing Teaching: Introduction to the Foundations of Education. New York: Allan \& Bacon.

Lather, P. (2007). Getting Lost: Feminist Practices. Toward a Double(d) Science. Albany, NY: SUNY Press.

Mayo, P. (2002). Public Pedagogy and the Quest for Substantive Democracy. Interchange, 33 (2), 193-207. Recuperado de http://www.springerlink.com/ content/4r8dre4pewve2ce $2 /$

Mayo, P. (2011). La pedagogia critica. Seminario corso di Dottorato di Ricerca. Universitá degli Studi di Verona, 27-28 Settembre. Recuperado de http:// www.google.co.cr/\#hl=en\&sclient=psyab\&q=Mayo $\% 2 \mathrm{C}+$ peter + pedagog $\%$ $\mathrm{C} 3 \% \mathrm{ACa}+$ critica\&oq=Mayo $\% 2 \mathrm{C}+$ peter+pedagog $\% \mathrm{C} 3 \% \mathrm{ACa}+$ critica\&gs_ $\mathrm{l}=-$ serp.3...3417.12961.0.15122.33.31.2.0.0.5.1691.15840.0j2j4j10j5j5j2j2j1.31.0...0.0...1c. ScjuLazuYkU\&pbx=1\&bav=on.2,or.r_gc.r_pw.r_qf.\&fp=5d6f9ca7f7e6cf2c\&bi$\mathrm{w}=1173 \& \mathrm{bih}=785$.

Masi, A. (2008). El concepto de praxis en Paulo Freire. En Gadotti, Moacir; Gómez, Margarita V., Mafra, Jason y Fernandes de Alencar, Ánderson (compiladores) Paulo Freire. Contribuciones para la pedagogía. Buenos Aires: CLACSO. Recuperado de http://www.campusvirtuales.com.ar/campusvirtuales/comun/mensajes/206273/1/Concepto\%20de\%20Praxis\%20en\%20Freire.pdf.

McLaren, P. (1988). On Ideology and Education. Critical pedagogy and the Politics of Empowerment.Recuperado de http://www.jstor.org/ discover $/ 10.2307 / 466183$ ?uid=2\&uid=4\&sid=21101195025377.

McLaren, P. (2005). Red Seminars: Radical Excursions into Educational Theory, Cultural Politics, and Pedagogy. Hampton Press.

McLaren, P. (2007). Life in schools: An Introduction to Critical Pedagogy in the Foundations of Education. Boston MA: Persons Allyn \& Bacon.

Meoño, R. (2011). Limitaciones que afectan a la Universidad Pública en el cumplimiento de sus funciones, derechos y responsabilidades. En curso de publicación.

O' Sullivan, E. (1999).Transformative learning. Educational Vision for the 21st. Century. London: Mcmillian.

Patel Stevens, L. \& Bean, T. W. (2007). Critical Literacy. Context, Research and Practice in the K-12 Classroom. Thousand Oaks, Ca: Sage.

Rodríguez Arocho, W. C. (2010). Pedagogía de la liberación y pedagogía crítica: un examen de sus aportes y retos. Pedagogía, 43 (1), 13-34. 
Rodríguez Arocho, W. C. (2008). La investigación educativa en América Latina:Cuatro desafíos y algunas ideas para enfrentarlos. Pedagogía, 41(1), 11-24.

Rossatto, C. A., Allen, R. L., \& Pruyn, M. (2006), (compiladores). Reinventing critical pedagogy. Widening the circle of anti-oppression education. Recuperado de http:// books.google.es/books?hl=es\&lr=\&id=tY $2 \mathrm{Ob} 3$ gRQC\&oi=fnd\&pg=PA125\&d$\mathrm{q}=\% 22 \mathrm{Patti}+$ Leather $\% 22+$ and + education\&ots $=$ kRwieUjpeo \&sig $=\mathrm{G} 58 \mathrm{Yb} 04 \mathrm{e}-$ 77EL11_LyUWxih4v3Rs\#v=onepage \&q=\%22Patti\%20Leather $\% 22 \% 20$ and $\% 20$ education \&f=false

Said, E. (1990).Orientalismo. Madrid: Libertarias.

Shor, I. \& Pari, C. (1999). Education is Politics. Critical Teaching across Differences, K-12 A Tribute to the life and Work of Paulo Freire.Manhattan Community College: CUNY.

Terigi, F. (2009). El fracaso escolar desde una perspectiva psicoeducativa: Hacia una reconceptualizaciòn situacional. Revista iberoamericana de educación, 50. Recuperado de http://www.rieoei.org/rie50a01.pdf.

Vilera, A. G. (2002). Reseña de pedagogía crítica y cultura depredadora. Políticas de oposición en la era posmoderna de Peter McLaren. Revista de Teoría y Didáctica de las Ciencias Sociales, 007, 271-275. Recuperado de http://redalyc. uaemex.mx/redalyc/pdf/652/65200713.pdf

Williams, R. (1983). Culture \& Society: 1790-1950. New York: Columbia University Press. 